\title{
Home Blood Pressure Measurements for Managing Hypertension in Hemodialysis Patients
}

\author{
Rajiv Agarwal ${ }^{a, b}$ Sangeetha Satyan ${ }^{a}$ Pooneh Alborzi ${ }^{a}$ Robert P. Light ${ }^{a}$

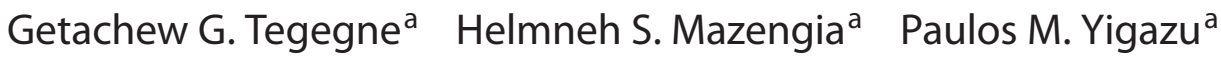 \\ ${ }^{a}$ Division of Nephrology, Indiana University School of Medicine, and ${ }^{b}$ Richard L. Roudebush VA Medical Center, \\ Indianapolis, Ind., USA
}

\section{Key Words}

Home blood pressure monitoring $\cdot$ Ambulatory blood

pressure monitoring $\cdot$ Hemodialysis $\cdot$ Hypertension

\begin{abstract}
Home blood pressure (BP) monitoring serves as a practical method to detect changes in BP instead of ambulatory BP monitoring in hemodialysis patients. To evaluate the relationship of reduction in home BP compared to interdialytic ambulatory BP measurements we analyzed the data from the dry-weight reduction in hypertensive hemodialysis patients (DRIP) trial in which 100 patients had their dry weight probed based on clinical sign and symptoms and 50 patients served as controls. We measured home BP 3 times a day for 1 week using a validated oscillometric monitor on 3 occasions at 4-week intervals after randomization. Changes from baseline in home, predialysis BP and postdialysis BP were compared to interdialytic 44-hour ambulatory BP. Home and ambulatory BP monitoring was available in 141 of 150 (94\%) patients. Predialysis systolic BP was not as sensitive as ambulatory BP in detecting change in BP with dry-weight reduction. Whereas postdialysis BP was capable of detecting an improvement in systolic BP in response to probing dry weight, by itself it does not provide evidence that change in postdialysis BP persists over the interdialytic period. Home $\mathrm{BP}$ reliably detected changes in ambulatory $\mathrm{BP}$, albeit with
\end{abstract}

less sensitivity at 4 weeks. However, at 4 and at 8 weeks, changes in home systolic BP were most strongly related to changes in interdialytic ambulatory systolic BP compared to predialysis and postdialysis $\mathrm{BP}$. The reproducibility of $\mathrm{BP}$ measurements followed the order home $>$ ambulatory $>>$ predialysis $>$ postdialysis. These data provide support for the use of home BP monitoring for the management of hypertension in hemodialysis patients.

Copyright $\odot 2009$ S. Karger AG, Basel

\section{Introduction}

Making a diagnosis of hypertension in patients on hemodialysis remains a matter of controversy [1-3]. The Kidney Disease Outcomes Quality Initiative (KDOQI) opinion-based guidelines recommend targeting predialysis blood pressure (BP) to $<140 / 90 \mathrm{~mm} \mathrm{Hg}$ and postdialysis BP to $<130 / 80 \mathrm{~mm} \mathrm{Hg}$ [4]. However, cohort studies suggest that when these targets are achieved, hemodialysis patients have more episodes of intradialytic hypotension compared to when these targets are not achieved [5]. Furthermore, there is only a weak relationship between predialysis and postdialysis BP measurements and interdialytic ambulatory $\mathrm{BP}$, such that predialysis or postdialysis BP measurements cannot be used to reliably predict interdialytic ambulatory $\mathrm{BP}[6,7]$.

\section{KARGER}

(C) 2009 S. Karger AG, Basel

Fax +4161306 1234

E-Mail karger@karger.ch

www.karger.com
Accessible online at:

www.karger.com/ajn
Prof. Rajiv Agarwal, MD

VAMC, $111 \mathrm{~N}, 1481$ West 10th St

Indianapolis, IN 46202 (USA)

Tel. +1 317988 2241, Fax +1 3179882171

E-Mail ragarwal@iupui.edu 
National and international guidelines call for monitoring home BP for the diagnosis and management of patients with hypertension $[8,9]$. These guidelines may be particularly relevant to hemodialysis patients because home BP monitoring is less expensive, less cumbersome, and more suited for long-term repeated use than interdialytic 44-hour ambulatory BP monitoring [10]. We have previously shown that $\mathrm{BP}$ obtained at home is a better determinant of interdialytic ambulatory BP compared to BP measurements obtained before and after dialysis [11]. Furthermore, home BP is a stronger determinant of left ventricular hypertrophy and all-cause mortality in hemodialysis patients compared to predialysis or postdialysis BP recordings $[12,13]$. These data are consistent with observations that home BP recordings in patients with chronic kidney disease but not on dialysis are a better marker of end-stage renal disease and all-cause mortality [14] and that home BP measurements obtained outside the clinic are better predictors of cardiovascular events in patients with essential hypertension $[15,16]$

Knowledge of the relationship of change in ambulatory, dialysis unit, and home BP measurements in response to deliberate intervention among hemodialysis patients is unknown. In the recently completed dryweight reduction in hypertensive hemodialysis patients (DRIP) trial we demonstrated a reduction of $\sim 7 / 3 \mathrm{~mm}$ $\mathrm{Hg}$ in response to probing dry weight measured by interdialytic ambulatory BP at 4 weeks which persisted at 8 weeks. The purpose of this report, a prespecified goal of this trial, was to evaluate the relationship of concurrent reduction in home $\mathrm{BP}$, predialysis $\mathrm{BP}$ and postdialysis $\mathrm{BP}$ compared to the gold standard of interdialytic ambulatory BP measurements.

\section{Methods}

The trial results and methods have been previously published [17]. Briefly, we recruited patients 18 years of age or older on longterm hemodialysis for at least 3 months, who had hypertension defined as mean interdialytic ambulatory BP of $135 / 85 \mathrm{~mm} \mathrm{Hg}$ or more. After a 6-hemodialysis run-in phase, during which baseline data were collected, patients were randomized in 1:2 proportion into control group versus ultrafiltration trial group for 8 weeks. During this 24-dialysis treatment phase, patients were seen at each dialysis visit and had dry weight probed as assessed by symptoms and signs related to hypovolemia $[18,19]$.

\section{BP Monitoring}

Ambulatory BP monitoring was performed after the midweek hemodialysis session for $44 \mathrm{~h}$. BP was recorded every $20 \mathrm{~min}$ during the day (6 a.m. to 10 p.m.) and every 30 min during the night (10 p.m. to 6 a.m.) using a Spacelab 90207 ABP monitor (SpaceLabs
Medical Inc., Redmond, Wash., USA) in the nonaccess arm. Recordings began immediately after hemodialysis and terminated immediately before the subsequent dialysis. Accuracy of ambulatory $\mathrm{BP}$ recordings was confirmed against auscultated BP at baseline. Hourly means were calculated. These means were then averaged over the entire course of recording to provide systolic and diastolic interdialytic ambulatory BPs.

Home BP monitoring was performed 3 times daily for 1 week. Patients were asked to record their BP in the morning, afternoon and before going to bed with a validated home BP monitor equipped with a memory device (HEM 705CP, Omron Healthcare, Bannockburn, Ill., USA).

Pre- and postdialysis BP measurements were recorded at each visit by the dialysis unit personnel using the oscillometric device equipped on the dialysis machine. No technique was specified to measure these BP. The mean of the last 3 treatments in the baseline period, the 3 treatments in week 4 and the 3 treatments at week 8 were taken to represent the pre- and postdialysis BP.

The study protocol was approved by the Institutional Review Boards (IRB) and the VA Research and Development Committee and all patients provided written informed consent. The trial was registered at ClinicalTrials.gov (NCT00067665).

\section{Data Extraction}

We studied only those patients with ambulatory BP recordings who also had home BP recordings and predialysis and postdialysis recordings. The last week of baseline, week 4 and week 8 predialysis and postdialysis BP measurements were averaged to obtain the mean predialysis and postdialysis BP at the end of each interval.

\section{Statistical Analysis}

We compared the change from baseline to 4 weeks in overall ambulatory $\mathrm{BP}$, home $\mathrm{BP}$, predialysis $\mathrm{BP}$ and postdialysis $\mathrm{BP}$ in the control and ultrafiltration groups. Next we compared the change from baseline to 8 weeks. The mean change between methods was compared using a mixed-effects model to account for repeated observations. We compared model fits to predict changes from baseline in ambulatory BP by regressing changes from baseline in predialysis, postdialysis and home BPs at 4 and 8 weeks and testing model fits using the Bayesian information criterion. The lower the Bayesian information criterion, the better is the model fit. The absolute difference between models was used to grade the evidence according to the following criteria: absolute difference $0-2$ (weak), 2-6 (positive), 7-10 (strong) and >10 (very strong) [20]. Separate models were fitted for systolic (SBP) and diastolic BP (DBP) recordings.

\section{Measurement of Reproducibility}

We compared the reproducibility from baseline to week 4 to week 8 within individuals for predialysis, postdialysis, home, and ambulatory BPs. We first fitted a mixed-effects model with full maximum likelihood estimates where the subject was the random variable and SBP was a fixed effect [21]. Specifically we fitted the following model $y_{i j}=\hat{\beta}+\zeta_{j}+\varepsilon_{i j}$, where $y_{i j}$ is the SBP for the $j$-th individual $(j=1, \ldots, N)$ with $i$-th measurement occasion $(i=$ $1, \ldots, 6), \zeta_{j}$ is the random intercept for the $j$-th individual (i.e., the individuals deviation from the overall mean), $\hat{\beta}$ is the estimated population mean SBP, and $\varepsilon_{i j}$ is the error for the $j$-th individual at the $i$-th measurement occasion. $\varepsilon_{i j}$ was assumed to distribute nor- 
mally and independently with a mean of zero and constant variance across individuals. This model provided the standard deviations between subjects and within subjects.

Next, the statistical significance of the reproducibility was tested by examining the residual variation in $\mathrm{BP}$ within individuals from one dialysis to the next. We constructed two mixed models. In the first model, SBP measured by a given method was used as a fixed effect and subject as the random effect. Specifically, the following model was fitted $y_{i j}=\hat{\beta}+\zeta_{j}+\varepsilon_{i j}$, where $y_{i j}$ is the SBP for the $j$-th individual $(j=1, \ldots, N)$ on the $i$-th measurement occasion $(j=1, \ldots, 6), \zeta_{j}$ is the random intercept for the $j$-th individual, and $\varepsilon_{i j}$ is the error for the $j$-th individual at the $i$-th measurement occasion. The second model contained SBP measured by the given method as well as ambulatory BP as a fixed effect as well as two nested random effects; one random effect was for subjects and another for the measurement method nested within subjects. This three-level model can be written as $y_{i j k}=\hat{\beta}+\zeta_{j k}+\zeta_{k}+\varepsilon_{i j k}$ where $\zeta_{j k}$ is the random intercept for method $\mathrm{j}$ and subject $\mathrm{k}$ and $\zeta_{k}$ is the random intercept for subject $\mathrm{k}$. The likelihood ratio test was used to compare the two nested models.

The nominal level of significance was set at two-sided $p<0.05$ and all statistical analyses were performed with Stata version 10.1 (StataCorp LP, College Station, Tex., USA).

\section{Results}

Five (10\%) patients in the control and $4(4 \%)$ in the ultrafiltration group had missing data. Of these, 9 patients had missing home BPs and 1 patient missing dialysis unit $\mathrm{BP}$ at baseline. At 4 weeks, 40 (80\%) patients in the control group and 85 (85\%) in the ultrafiltration group had complete data for all BP measurement modalities. At 8 weeks, 39 (78\%) patients in the control group and 86 $(86 \%)$ in the ultrafiltration group had complete data. These data form the basis of the current analysis.

The baseline characteristics of the patients eligible at baseline for this analysis are shown in table 1. The two treatment groups were well balanced with respect to the baseline characteristics of the patients.

Table 2 shows the SBP obtained before and after dialysis, at home by the patients through self-measurement and by ambulatory BP monitoring. In the ultrafiltration group, predialysis SBP declined $8.0 \mathrm{~mm} \mathrm{Hg}$ at 4 weeks and $11.7 \mathrm{~mm} \mathrm{Hg}$ at 8 weeks. Although these changes were significant from baseline, when adjusted for changes from baseline in the control group, the ultrafiltration-attributable effect on predialysis SBP was not significant at 4 weeks, but was at 8 weeks.

Postdialysis SBP had a response that was different from predialysis SBP. Although in the ultrafiltration group postdialysis SBP declines were comparable to that seen with predialysis SBP, the control group had a smaller change at 4 weeks and an insignificant increase was
Table 1. Clinical characteristics of the study population

\begin{tabular}{|c|c|c|}
\hline Clinical characteristic & $\begin{array}{l}\text { Ultrafiltration } \\
(\mathrm{n}=96)\end{array}$ & $\begin{array}{l}\text { Control } \\
(\mathrm{n}=45)\end{array}$ \\
\hline Age, years & $54.2 \pm 12.9$ & $54.0 \pm 12.1$ \\
\hline Men & $63(65 \%)$ & $34(76 \%)$ \\
\hline \multicolumn{3}{|l|}{ Race } \\
\hline White & $12(13 \%)$ & $2(4 \%)$ \\
\hline Black & $81(84 \%)$ & $42(93 \%)$ \\
\hline Other & $3(3 \%)$ & $1(2 \%)$ \\
\hline Predialysis weight, $\mathrm{kg}$ & $83.7 \pm 19.3$ & $84.9 \pm 20.5$ \\
\hline Postdialysis weight, kg & $80.7 \pm 18.7$ & $82.1 \pm 19.9$ \\
\hline Body mass index & $27.3 \pm 5.8$ & $27.2 \pm 6.6$ \\
\hline Years of dialysis & $3.9 \pm 4.8$ & $4.7 \pm 6.0$ \\
\hline \multicolumn{3}{|c|}{ Etiology of end-stage renal disease } \\
\hline Diabetes mellitus & $37(38 \%)$ & $17(38 \%)$ \\
\hline Hypertension & $46(47 \%)$ & $22(49 \%)$ \\
\hline Glomerulonephritis & $4(4 \%)$ & $1(2 \%)$ \\
\hline Polycystic kidney disease & $3(3 \%)$ & $0(0 \%)$ \\
\hline Other & $6(6 \%)$ & $5(11 \%)$ \\
\hline Current smokers & $31(32 \%)$ & $16(36 \%)$ \\
\hline \multicolumn{3}{|l|}{ History of } \\
\hline Congestive heart failure & $16(17 \%)$ & $3(7 \%)$ \\
\hline Myocardial infarction & $14(15 \%)$ & $5(11 \%)$ \\
\hline Stroke & $9(9 \%)$ & $4(9 \%)$ \\
\hline Urea reduction ratio & $74.3 \pm 6.8$ & $73.5 \pm 6.4$ \\
\hline Albumin, g/dl & $3.7 \pm 0.5$ & $3.7 \pm 0.4$ \\
\hline Hemoglobin, g/dl & $12.2 \pm 1.1$ & $12.0 \pm 1.3$ \\
\hline Presence of edema ${ }^{1}$ & $18(19 \%)$ & $6(13 \%)$ \\
\hline \multicolumn{3}{|l|}{ Number receiving } \\
\hline antihypertensive drugs & $83(86 \%)$ & $35(78 \%)$ \\
\hline \multicolumn{3}{|l|}{ Number of antihypertensives } \\
\hline in users & $2.7 \pm 1.4$ & $2.6 \pm 1.3$ \\
\hline
\end{tabular}

${ }^{1}$ Missing in 2 patients in the ultrafiltration group.

seen at 8 weeks. The ultrafiltration-attributable effects on postdialysis SBP were significant at 4 and 8 weeks.

On average about $13-15$ readings were available per week for home BP. The changes in control group from baseline group were $<2 \mathrm{~mm} \mathrm{Hg}$ at 4 and 8 weeks. In contrast, BPs of the ultrafiltration group declined significantly by $6.5 \mathrm{~mm} \mathrm{Hg}$ at 4 weeks and $11.8 \mathrm{~mm} \mathrm{Hg}$ at 8 weeks. The ultrafiltration-attributable decline in home SBP was marginally significant at 4 weeks and highly significant at 8 weeks. The decline in home SBP from 4 to 8 weeks was $7.8 \mathrm{~mm} \mathrm{Hg}$, which was statistically significant $(\mathrm{p}=0.013)$.

On average about $80-90$ readings were available per recording for ambulatory BP. BP decline in the control group at 8 weeks from baseline of $5.4 \mathrm{~mm} \mathrm{Hg}$ was statistically significant. The ultrafiltration group experienced 
Table 2. Systolic ambulatory, home and dialysis unit blood pressure

\begin{tabular}{|c|c|c|c|}
\hline BP component & Baseline & $\begin{array}{l}\text { Change at } 4 \text { weeks } \\
\text { from baseline }\end{array}$ & $\begin{array}{l}\text { Change at } 8 \text { weeks } \\
\text { from baseline }\end{array}$ \\
\hline \multicolumn{4}{|l|}{ Predialysis } \\
\hline Control $(95 \% \mathrm{CI})$ & $161.1(155.7$ to 166.4$)$ & $-3.6(-9.2$ to 2.0$)$ & $-3.8(-9.4$ to 1.9$)$ \\
\hline UF (95\% CI) & $159.7(156.1$ to 163.4$)$ & $-8.0(-11.8 \text { to }-4.1)^{\mathrm{c}}$ & $-11.7(-15.5 \text { to }-7.8)^{\mathrm{c}}$ \\
\hline Difference between groups, UF - control (95\% CI) & $-1.3(-7.8$ to 5.2$)$ & $-4.4(-11.2$ to 2.4$)$ & $-7.9(-14.8 \text { to }-1.0)^{\mathrm{a}}$ \\
\hline $\mathrm{p}$ value of difference between groups & $>0.2$ & $>0.2$ & 0.02 \\
\hline \multicolumn{4}{|l|}{ Postdialysis } \\
\hline Control $(95 \% \mathrm{CI})$ & $143.4(137.7$ to 149.1$)$ & $0.6(-5.4$ to 6.6$)$ & $4.2(-1.8$ to 10.3$)$ \\
\hline $\mathrm{UF}(95 \% \mathrm{CI})$ & $143.7(139.8$ to 147.6$)$ & $-8.6(-12.8 \text { to }-4.5)^{\mathrm{c}}$ & $-10.5(-14.6 \text { to }-6.3)^{c}$ \\
\hline Difference between groups, UF - control (95\% CI) & $0.3(-6.6$ to 7.2$)$ & $-9.3(-16.5 \text { to }-2.0)^{\mathrm{b}}$ & $-14.7(-22.0 \text { to }-7.4)^{c}$ \\
\hline $\mathrm{p}$ value of difference between groups & $>0.2$ & 0.01 & 0.001 \\
\hline \multicolumn{4}{|l|}{ Home } \\
\hline Number of readings, control $( \pm S D)$ & $13 \pm 4$ & $15 \pm 4$ & $15 \pm 4$ \\
\hline Control $(95 \% \mathrm{CI})$ & $158.0(152.6$ to 163.5$)$ & $-1.0(-5.9$ to 3.9$)$ & $1.5(-3.5$ to 6.4$)$ \\
\hline Number of readings, UF $( \pm S D)$ & $13 \pm 5$ & $14 \pm 4$ & $14 \pm 5$ \\
\hline UF $(95 \%$ CI) & $159.3(155.5$ to 163.0$)$ & $-6.5(-9.9 \text { to }-3.1)^{\mathrm{c}}$ & $-11.8(-15.1 \text { to }-8.4)^{\mathrm{c}}$ \\
\hline Difference between groups, UF - control (95\% CI) & $1.2(-5.3$ to 7.8$)$ & $-5.5(-11.4$ to 0.4$)$ & $-13.3(-19.2 \text { to }-7.3)^{\mathrm{c}}$ \\
\hline $\mathrm{p}$ value of difference between groups & $>0.2$ & 0.07 & $<0.001$ \\
\hline \multicolumn{4}{|l|}{ 44-hour interdialytic } \\
\hline Number of readings, control $( \pm S D)$ & $90 \pm 23$ & $87 \pm 24$ & $80 \pm 23$ \\
\hline Control $(95 \% \mathrm{CI})$ & $146.5(142.3$ to 150.8$)$ & $-2.6(-7.3$ to 2.2$)$ & $-5.4(-10.2 \text { to }-0.6)^{\mathrm{a}}$ \\
\hline Number of readings, UF $( \pm S D)$ & $90 \pm 22$ & $89 \pm 21$ & $83 \pm 28$ \\
\hline UF $(95 \%$ CI $)$ & $145.7(142.8$ to 148.6$)$ & $-10.6(-13.9 \text { to }-7.3)^{\mathrm{c}}$ & $-13.7(-17.0 \text { to }-10.4)^{\mathrm{c}}$ \\
\hline Difference between groups, UF - control (95\% CI) & $-0.9(-6.0$ to 4.3$)$ & $-8(-13.8 \text { to }-2.2)^{\mathrm{b}}$ & $-8.3(-14.1 \text { to }-2.5)^{\mathrm{b}}$ \\
\hline $\mathrm{p}$ value of difference between groups & $>0.2$ & 0.01 & 0.01 \\
\hline
\end{tabular}

$0.01<{ }^{\mathrm{a}} \mathrm{p}<0.05 ; 0.001<{ }^{\mathrm{b}} \mathrm{p}<0.01 ;{ }^{\mathrm{c}} \mathrm{p}<0.001 . \mathrm{UF}=$ Ultrafiltration.

highly significant reductions in SBP of $10.6 \mathrm{~mm} \mathrm{Hg}$ at 4 weeks and $13.7 \mathrm{~mm} \mathrm{Hg}$ at 8 weeks. The ultrafiltration-attributable decline in 44-hour interdialytic ambulatory $\mathrm{SBP}$ was $8.0 \mathrm{~mm} \mathrm{Hg}$ at 4 weeks and $8.3 \mathrm{~mm} \mathrm{Hg}$ at 8 weeks. No additional reduction in ambulatory $\mathrm{BP}$ was seen from 4 to 8 weeks either in the ultrafiltration group or in the control group.

Figure 1 illustrates the comparative changes in SBP from baseline in the ultrafiltration group minus the control group. The error bars represent the $95 \%$ confidence interval of the change and when the upper limit of the confidence interval traverses the zero line, the statistical significance is lost. The most consistent reduction in SBP was seen in the ambulatory BP group. The predialysis SBP was reduced at 8 weeks when only the paired data are considered, but when all available data were analyzed (fig. 1b), the statistical significance of the change in predialysis SBP was lost. Postdialysis SBP was reduced at 4 and 8 weeks. Home SBP was reduced at 8 weeks and marginally at 4 weeks.

The changes in DBP were directionally and qualitatively similar to those seen with SBP (data not shown).

The degree of model fit was compared for various regression models predicting the change in ambulatory $\mathrm{BP}$ by calculating the difference in the Bayesian information criterion of the models (table 3). The best predictor of change in ambulatory $\mathrm{BP}$ was the change in home SBP and not predialysis or postdialysis $\mathrm{BP}$ whether the change was calculated at 4 or 8 weeks. DBP change was best predicted by change in predialysis DBP at 4 weeks and postdialysis DBP at 8 weeks. However, at 8 weeks home BP was similar to postdialysis DBP in predicting change in ambulatory BP.

Figure 2 shows a dot plot that illustrates SBP at baseline visit in the control and ultrafiltration groups. Ambulatory BP had the least variability between subjects. The 


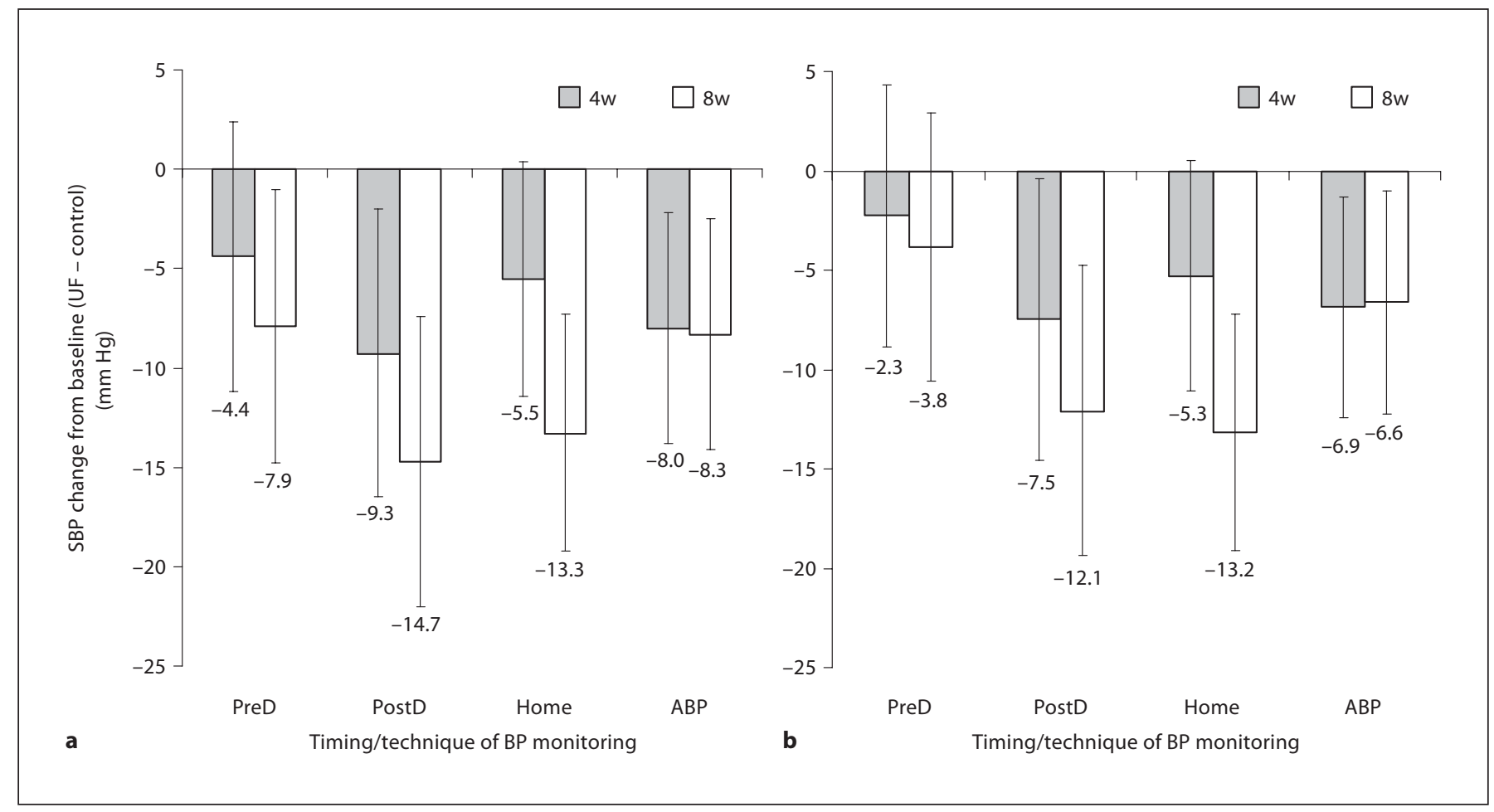

Fig. 1. Mean SBP changes from baseline to 4 weeks (4w) and from baseline to 8 weeks $(8 \mathrm{w})$ in the ultrafiltration (UF) group minus changes from baseline to 4 weeks and from baseline to 8 weeks in the control group. The error bars are the $95 \%$ confidence intervals of the means. When the bars transect the zero line, then the changes from baseline are not statistically significant. a The paired data shown are those detailed in table 2. b All available data. Predialysis (PreD) BP is insensitive in detecting change. Post $\mathrm{D}=$ Postdialysis; $\mathrm{ABP}=$ ambulatory BP. bottom panel shows the standard deviation within individuals from baseline to 4 weeks to 8 weeks which illustrates the time-dependent variation. The residual variation in SBP was $14.8 \mathrm{~mm} \mathrm{Hg}$ for postdialysis BP, $14.0 \mathrm{~mm}$ $\mathrm{Hg}$ for predialysis $\mathrm{BP}, 12.4 \mathrm{~mm} \mathrm{Hg}$ for home $\mathrm{BP}$ and 12.6 $\mathrm{mm} \mathrm{Hg}$ for ambulatory BP. Home and ambulatory BP were both more reproducible compared to predialysis and postdialysis BP (likelihood ratio test $<0.001$ for all comparisons). Home BP was more reproducible compared to ambulatory BP (likelihood ratio test $=0.02$ ).

\section{Discussion}

The main findings of the present study are: (1) the changes in response to nonpharmacological intervention in hemodialysis patients with hypertension can be most reliably detected by 44-hour interdialytic ambulatory BP monitoring as early as after 4 weeks, (2) predialysis SBP is not as sensitive as ambulatory BP in detecting this change, (3) while postdialyis BP is capable of detecting an improvement in SBP in response to a nonpharmacological intervention, it instills little confidence that such changes persist over the interdialytic period, (4) home BP can reliably detect changes in $\mathrm{BP}$, but home $\mathrm{BP}$ is not as sensitive as ambulatory BP monitoring in detecting early changes (within 4 weeks) with intervention, (5) changes in home SBP were most strongly related to changes in interdialytic ambulatory SBP, and (6) ambulatory and home $\mathrm{BP}$ were more reproducible, i.e. they had greater test-retest reliability compared to predialysis and postdialysis BP.

It is now well recognized that ambulatory $\mathrm{BP}$ monitoring is more precise in detecting changes in $\mathrm{BP}$ among individuals $[22,23]$. However, these data have been largely generated in patients who are not on hemodialysis. Hemodialysis can cause large changes in volume and may disturb circadian rhythms which can be captured by the 
Table 3. Relationships between changes in ambulatory BP, dialysis unit BP and home BP

\begin{tabular}{|c|c|c|c|c|c|c|}
\hline \multirow[t]{2}{*}{ Timing of $\mathrm{BP}$} & \multicolumn{3}{|c|}{ SBP beta coefficients ( 4 weeks) } & \multicolumn{3}{|c|}{ SBP beta coefficients ( 8 weeks) } \\
\hline & preHD & postHD & home & preHD & postHD & home \\
\hline \multirow{8}{*}{$\begin{array}{l}\text { Coefficient } \\
\text { Constant } \\
\mathrm{r}^{2} \\
\text { RMSE } \\
\text { AIC } \\
\text { BIC } \\
\text { Extent of evidence for home BP as } \\
\text { a better model vs. model in column }\end{array}$} & 0.258 & 0.219 & 0.371 & 0.410 & 0.356 & 0.501 \\
\hline & -6.5 & -6.8 & -6.4 & -6.9 & -8.6 & -7.0 \\
\hline & 0.103 & 0.078 & 0.123 & 0.212 & 0.246 & 0.298 \\
\hline & 14.13 & 14.32 & 13.96 & 15.36 & 15.03 & 14.50 \\
\hline & $1,002.5$ & $1,005.8$ & 999.6 & $1,014.8$ & $1,009.5$ & $1,006.3$ \\
\hline & $1,008.1$ & $1,011.4$ & $1,005.2$ & $1,020.4$ & $1,015.1$ & $1,000.7$ \\
\hline & positive & strong & & very strong & strong & \\
\hline & \multicolumn{3}{|c|}{ DBP beta coefficients ( 4 weeks) } & \multicolumn{3}{|c|}{ DBP beta coefficients ( 8 weeks) } \\
\hline Parameter: & preHD & postHD & home & preHD & postHD & home \\
\hline Slope & 0.359 & 0.305 & 0.521 & 0.302 & 0.304 & 0.434 \\
\hline Constant & -3.7 & -4.8 & -3.7 & -4.6 & -4.8 & -4.4 \\
\hline $\mathrm{r}^{2}$ & 0.203 & 0.227 & 0.155 & 0.156 & 0.227 & 0.219 \\
\hline RMSE & 7.9 & 7.7 & 8.1 & 8.1 & 7.7 & 7.8 \\
\hline AIC & 858.3 & 873.6 & 865.7 & 858.2 & 847.3 & 848.7 \\
\hline BIC & 864.0 & 879.2 & 871.3 & 863.8 & 852.9 & 854.3 \\
\hline $\begin{array}{l}\text { Extent of evidence for home BP as } \\
\text { a better model vs. model in column }\end{array}$ & $\begin{array}{l}\text { home worse } \\
\text { (strong) }\end{array}$ & strong & & strong & $\begin{array}{l}\text { home worse } \\
\text { (weak) }\end{array}$ & \\
\hline
\end{tabular}

RMSE $=$ Root mean squared error; AIC $=$ Akaike information criterion; BIC $=$ Bayesian information criterion.

technique of ambulatory BP monitoring [24]. While the greater number of measurements is undoubtedly important in obtaining a more reliable mean, it is also possible that sampling BP outside the dialysis unit is better related to the health of the arterial tree and long-term outcomes $[13,25,26]$. Although ambulatory BP is an excellent research tool, it is cumbersome and difficult to use on a large scale for the diagnosis and treatment of hypertension in hemodialysis patients. Home BP monitoring better relates with ambulatory BP, but its ability to detect longitudinal changes in $\mathrm{BP}$ among hemodialysis patients was unclear prior to this report. Our data provide evidence that home $\mathrm{BP}$ can detect changes in ambulatory $\mathrm{BP}$ and that it is more strongly related to change in ambulatory $\mathrm{BP}$ compared to predialysis or postdialysis measurements. Furthermore, our report extends the observations of reproducibility of ambulatory BP measurements in hemodialysis patients to BP measurements obtained at home [27].

In their review, Thompson and Pickering [1] suggest that ambulatory or some form of home BP monitoring should be more widely adopted for patients with end- stage renal disease. Their recommendations are further strengthened by two published reports utilizing home BP monitoring to detect changes in BP in hemodialysis patients. Kauric-Klein and Artinian [28] randomized 17 chronic hemodialysis patients to receive home BP monitor intervention in addition to usual care, whereas an additional 17 participants were randomized to usual care without home BP monitoring. Significant reductions in systolic average weekly home BP measurements were seen in the home BP group. No significant differences were found between groups in terms of $\mathrm{DBP}$ or interdialytic weight gains. These findings indicate that home BP monitoring per se could help improve BP control in the hemodialysis population. Another 6-month randomized, controlled trial tested the notion that the automatic feedback system of dialysis will improve hypertension control compared with standard dialysis [29]. A systematic BP management protocol was used in addition to the automatic feedback system dialysis or standard dialysis in all patients. A novelty of this study conducted in 44 longterm hemodialysis patients was the use of home BP measurement as a primary endpoint. The intervention groups 


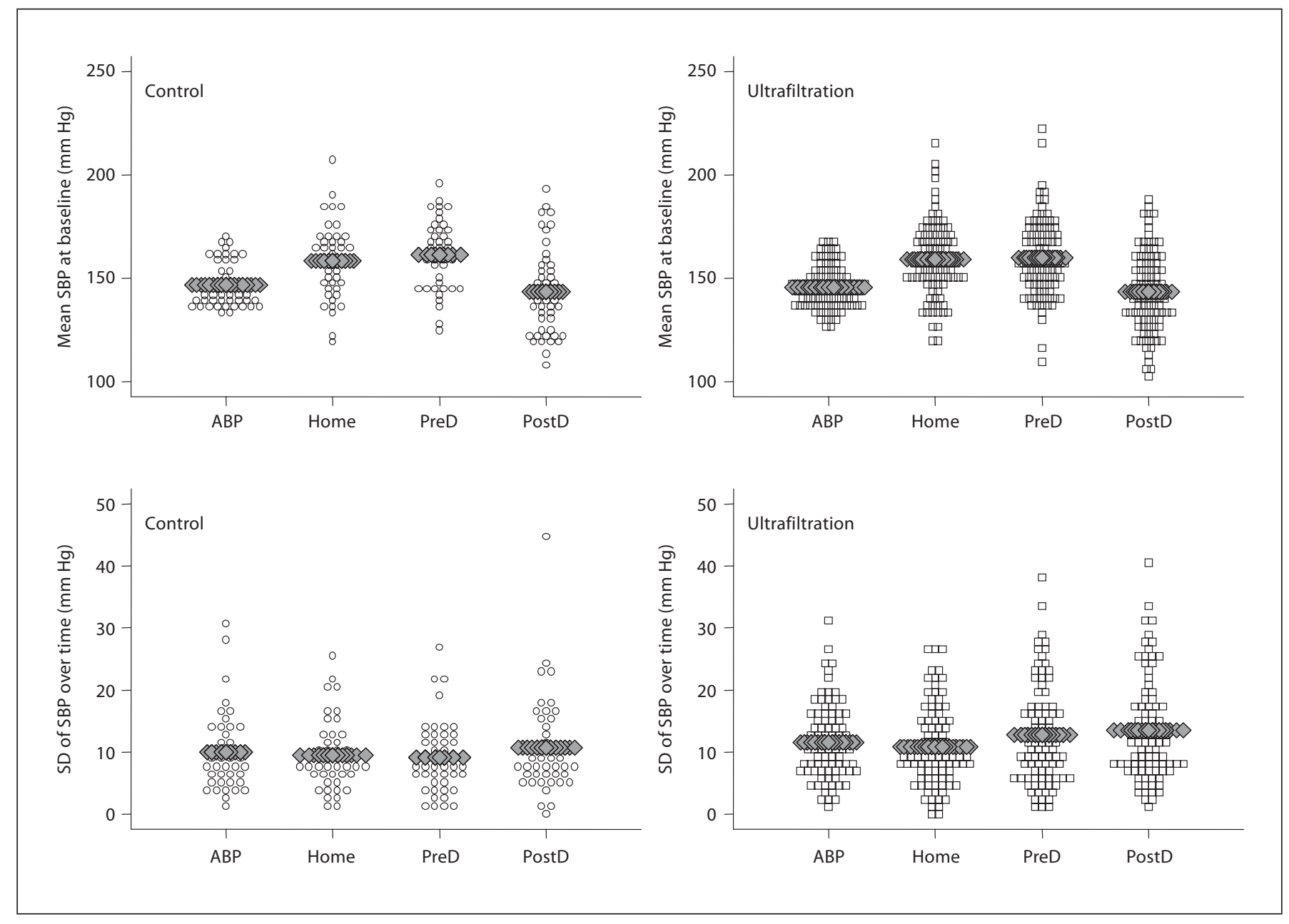

Fig. 2. Mean $\mathrm{SBP}$ at baseline in the control and ultrafiltration groups shows that ambulatory BP (ABP) has the least interindividual variation. The mean level of home $B P$ is higher than ambulatory BP. Intraindividual changes in SBP in the bottom panel show that home BP has the lowest standard deviation. Predialysis (preD) and postdialysis (postD) BP were less reproducible than home or ambulatory BP. In our study, the test-retest reliability of home BP was the greatest. See text for details. had a drop in SBP from 147.8 to $139.8 \mathrm{~mm} \mathrm{Hg}$ and the control group from 141.9 to $135.2 \mathrm{~mm} \mathrm{Hg}(\mathrm{p}=0.005$ for change from baseline). The authors were able to detect a remarkable improvement in SBP during the study period despite a limited number of patients.

In a recent meta-analysis, Ishikawa et al. [30] reported that changes in home $\mathrm{BP}$ in response to antihypertensive therapy are slightly less than those seen with clinic BP, but slightly greater than those recorded by ambulatory $\mathrm{BP}$ recordings. Although they excluded hemodialysis patients from their meta-analysis, we note that the changes in home $\mathrm{BP}$ are less than postdialysis $\mathrm{BP}$. The changes in interdialytic ambulatory $\mathrm{BP}$ are the least of all. However, interdialytic ambulatory $\mathrm{BP}$ monitoring is able to detect smaller changes in $\mathrm{BP}$ given its greater precision. It is notable that at 4 weeks, ambulatory BP could detect a change that was not possible with predialysis or home BP. Although this change could be detected by postdialysis BP, the clinical significance of this change is questionable given the nature of the study design. Since we were probing dry weight, it would be of no surprise that the intervention group would have a lower $\mathrm{BP}$ compared to the control group. However, in the absence of interdialytic measurements, persistent decline in interdialytic BP would be questionable.

A limitation of our study is that it was overrepresented by black hemodialysis patients. Whether black participants have a different response to home BP than nonblack 
participants is not known. However, greater representation by nonblack participants would have made the study more generalizable. A strength of our study was the use of a validated home BP monitor which was equipped with a memory device and all participants were instructed in its use. Ambulatory BP monitoring and simultaneous home BP monitoring make this study unique in being the first in hemodialysis patients utilizing both methods of BP measurement.

Recent American Heart Association guidelines call for home BP monitoring in children, the elderly, pregnant women, patients with chronic kidney disease or diabetes mellitus and for the detection and treatment of essential hypertension. These data provide support for the role of home BP monitoring for the management of hypertension in hemodialysis patients. They challenge the notion that for practical and financial reasons, self-measured home BP cannot be applied to the totality of dialysis patients [4]. It is time that home BP monitoring was more widely applied to the diagnosis and management of hypertension in hemodialysis patients [31].

\section{Acknowledgments}

This study was supported by grant number 5RO1-NIDDK062030-05 from National Institutes of Health.

We are indebted to the participating hemodialysis patients who volunteered their time; staff of the dialysis units at Dialysis Clinics, Inc., Clarian Health, and the Roudebush VA Medical Center; the faculty of the Division of Nephrology for allowing us to study their patients; the research technicians, research fellows and to the members of the Data and Safety Monitoring Board.

\section{References}

1 Thompson AM, Pickering TG: The role of ambulatory blood pressure monitoring in chronic and end-stage renal disease. Kidney Int 2006;70:1000-1007.

2 Lazar AE, Smith MC, Rahman M: Blood pressure measurement in hemodialysis patients. Semin Dial 2004;17:250-254.

-3 Agarwal R: Assessment of blood pressure in hemodialysis patients. Semin Dial 2002;15: 299-304.

$\checkmark 4 \mathrm{~K} / \mathrm{DOQI}$ clinical practice guidelines for cardiovascular disease in dialysis patients. Am J Kidney Dis 2005;45:S1-S153.

5 Davenport A, Cox C, Thuraisingham R: Achieving blood pressure targets during dialysis improves control but increases intradialytic hypotension. Kidney Int 2007;73: 759-764.

6 Agarwal R, Peixoto AJ, Santos SF, Zoccali C: Pre- and postdialysis blood pressures are imprecise estimates of interdialytic ambulatory blood pressure. Clin J Am Soc Nephrol 2006; 1:389-398.

7 Agarwal R, Metiku T, Tegegne GG, Light RP, Bunaye Z, Bekele DM, Kelley K: Diagnosing hypertension by intradialytic blood pressure recordings. Clin J Am Soc Nephrol 2008;3: 1364-1372.

-8 Pickering TG, Miller NH, Ogedegbe G, Krakoff LR, Artinian NT, Goff D: Call to action on use and reimbursement for home blood pressure monitoring: a joint scientific statement from the American Heart Association, American Society of Hypertension, and Preventive Cardiovascular Nurses Association. Hypertension 2008;52:10-29.
Parati G, Stergiou GS, Asmar R, Bilo G, de Leeuw P, Imai Y, Kario K, Lurbe E, Manolis A, Mengden T, O’Brien E, Ohkubo T, Padfield P, Palatini P, Pickering T, Redon J, Revera M, Ruilope LM, Shennan A, Staessen JA, Tisler A, Waeber B, Zanchetti A, Mancia G: European Society of Hypertension guidelines for blood pressure monitoring at home: a summary report of the Second International Consensus Conference on Home Blood Pressure Monitoring. J Hypertens 2008;26: 1505-1526.

10 Agarwal R: Role of home blood pressure monitoring in hemodialysis patients. Am J Kidney Dis 1999;33:682-687.

11 Agarwal R, Andersen MJ, Bishu K, Saha C: Home blood pressure monitoring improves the diagnosis of hypertension in hemodialysis patients. Kidney Int 2006;69:900-906.

12 Agarwal R, Brim NJ, Mahenthiran J, Andersen MJ, Saha C: Out-of-hemodialysis-unit blood pressure is a superior determinant of left ventricular hypertrophy. Hypertension 2006;47:62-68.

13 Alborzi P, Patel N, Agarwal R: Home blood pressures are of greater prognostic value than hemodialysis unit recordings. Clin J Am Soc Nephrol 2007;2:1228-1234.

14 Agarwal R, Andersen MJ: Prognostic importance of clinic and home blood pressure recordings in patients with chronic kidney disease. Kidney Int 2006;69:406-411.

15 Bobrie G, Chatellier G, Genes N, Clerson P, Vaur L, Vaisse B, Menard J, Mallion JM: Cardiovascular prognosis of 'masked hypertension' detected by blood pressure self-measurement in elderly treated hypertensive patients. JAMA 2004;291:1342-1349.
16 Ohkubo T, Imai Y, Tsuji I, Nagai K, Kato J, Kikuchi N, Nishiyama A, Aihara A, Sekino M, Kikuya M, Ito S, Satoh H, Hisamichi S: Home blood pressure measurement has a stronger predictive power for mortality than does screening blood pressure measurement: a population-based observation in Ohasama, Japan. J Hypertens 1998;16:971-975.

17 Agarwal R, Alborzi P, Satyan S, Light RP: Dry-weight reduction in hypertensive hemodialysis patients. A randomized, controlled trial. Hypertension 2009;53:500507.

18 Charra B, Laurent G, Chazot C, Calemard E, Terrat JC, Vanel T, Jean G, Ruffet M: Clinical assessment of dry weight. Nephrol Dial Transplant 1996;11(suppl 2):16-19.

19 Jaeger JQ, Mehta RL: Assessment of dry weight in hemodialysis: an overview. J Am Soc Nephrol 1999;10:392-403.

20 Singer JD, Willett JB: Applied Longitudinal Data Analysis: Modeling Change and Event Occurrence. Oxford, Oxford University Press, 2003.

21 Holden JE, Kelley K, Agarwal R: Analyzing change: a primer on multilevel models with applications to nephrology. Am J Nephrol 2008;28:792-801

22 Pickering TG, Shimbo D, Haas D: Ambulatory blood-pressure monitoring. N Engl J Med 2006;354:2368-2374.

$\checkmark 23$ Peixoto AJ, White WB: Ambulatory blood pressure monitoring in chronic renal disease: technical aspects and clinical relevance. Curr Opin Nephrol Hypertens 2002;11:507516. 
-24 Kelley K, Light RP, Agarwal R: Trended cosinor change model for analyzing hemodynamic rhythm patterns in hemodialysis patients. Hypertension 2007;50:143-150.

25 Agarwal R, Andersen MJ, Light RP: Location not quantity of blood pressure measurements predicts mortality in hemodialysis patients. Am J Nephrol 2007;28:210-217.

-26 Agarwal R, Light RP: Arterial stiffness and interdialytic weight gain influence ambulatory blood pressure patterns in hemodialysis patients. Am J Physiol Renal Physiol 2007; 294:F303-F308.
27 Peixoto AJ, Santos SF, Mendes RB, Crowley ST, Maldonado R, Orias M, Mansoor GA, White WB: Reproducibility of ambulatory blood pressure monitoring in hemodialysis patients. Am J Kidney Dis 2000;36:983990.

28 Kauric-Klein Z, Artinian N: Improving blood pressure control in hypertensive hemodialysis patients. CANNT J 2007;17:2426.

29 Deziel C, Bouchard J, Zellweger M, Madore F: Impact of hemocontrol on hypertension, nursing interventions, and quality of life: a randomized, controlled trial. Clin J Am Soc Nephrol 2007;2:661-668.
30 Ishikawa J, Carroll DJ, Kuruvilla S, Schwartz JE, Pickering TG: Changes in home versus clinic blood pressure with antihypertensive treatments: a meta-analysis. Hypertension 2008;52:856-864.

31 Agarwal R: How should hypertension be assessed and managed in hemodialysis patients? Home BP, not dialysis unit BP, should be used for managing hypertension. Semin Dial 2007;20:402-405. 